\title{
Cancer-Associated-Fibroblast Induces Epithelial-Mesenchymal Transition of Gastric Cancer Cells via Activating Thy-1
}

\author{
Weiguo $\mathrm{Hu}^{1,2 \#}$, Chao $\mathrm{Li}^{1,2 \#}$, Jing Sun ${ }^{1,2}$, Bo Feng ${ }^{1,2}$, Daohai Zhang ${ }^{3}$, Junjun $\mathrm{Ma}^{1,2}$, Lu Zang ${ }^{1,2}$, Hongchao Zhao ${ }^{1,2}$, Weiguo Xu ${ }^{1,2}$, Binya Liu ${ }^{4}$, \\ Minhua Zheng ${ }^{1,2 * \#}$ and Zhenggang Zhu ${ }^{1,2 *}$
}

${ }^{1}$ Department of General Surgery, Ruijin Hospital, Shanghai Jiao Tong University School of Medicine, Shanghai, P. R. China

${ }^{2}$ Shanghai Minimally Invasive Surgery Center, Shanghai, P. R. China

${ }^{3}$ Cancer Research Group, The Canberra Hospital, and ANU Medical School, The Australia National University, Garran, ACT, Australia

${ }^{4}$ Shanghai Institute of Digestive Surgery, Ruijin Hospital, Shanghai Jiao Tong University School of Medicine, Shanghai, P. R. China

\#Contributed equally as co-first authors.

\begin{abstract}
Cancer-associated fibroblasts (CAFs) are the key stromal cells in solid tumor microenvironment and are crucial for facilitating tumor metastasis. However, the detailed mechanism of how CAFs induce metastasis in human gastric cancer remains to be elucidated. In this study, using the CAFs isolated from human gastric tumor tissues, we have demonstrated that co-culturing of the CAFs with gastric cancer cells could activate the epithelial-mesenchymal transition (EMT) program, leading to enhanced cancer cell migration and invasion. In the CAF cells, Thy- 1 expression was significantly increased, compared to the normal fibroblasts. Mechanistic studies revealed that depletion of Thy1 by siRNA in CAF cells attenuated the CAFs-induced EMT and aggressiveness in gastric cancer cells. Taken together, our studies indicate a significant role of Thy- 1 in CAFs-induced gastric cancer progression. Targeting Thy-1 could be a potential therapeutic strategy for gastric cancer treatment.
\end{abstract}

Keywords: Cancer-associated fibroblast; Gastric cancer; Metastasis; Epithelial-mesenchymal transition; Thy-1

\section{Introduction}

Despite standardizations and breakthroughs in comprehensive therapy, gastric cancer is one of the major causes of cancer-related mortality, especially in East Asia [1-3]. Thus, more efforts have been made to understand the mechanisms underlying the gastric cancer progression, among which the tumor microenvironment is one of the priorities of research [4-6]. As one of the key stromal cells in solid tumor microenvironment, cancer-associated fibroblasts (CAFs) can be identified from other stromal cells by examining their phenotypic characteristics such as the typical spindle shape, and the molecular biomarkers including fibroblast-specific protein 1 (FSP-1), $a$-smooth muscle actin ( $\alpha$-SMA), vimentin, desmin, type I collagen, plateletderived growth factor receptor-B (PDGFR $\beta$ ) and fibroblast-activated protein (FAP) [7-9]. CAFs have been reported to play a crucial role in metastasis of human solid tumors originated from stomach [10-13], colon [14-17], breast [18-21], prostate [22,23], pancreas [24,25] and so on. However, the underlying mechanisms of the CAFs-induced metastasis in human solid tumors are still unclear and need to be investigated.

Unlike a basic physiological phenomenon in human embryonic development, the theory of epithelial-mesenchymal transition (EMT) in tumor progression has been regarded as one of the classic mechanisms of tumor migration, invasion and metastasis [26]. During the EMT process, tumor epithelial cells acquire the typical mesenchymal traits: for instance, the fibroblast-like phenotype, destruction of cell-cell tight junction, attenuation of cell adhesion and activation of cell moveability [27-29]. Accordingly, the EMT in tumor progression has been characterized by the alterations of several biomarkers including reduction of epithelial marker E-cadherin and increase of mesenchymal markers including $\mathrm{N}$-cadherin, vimentin and fibronectin [27]. Moreover, several key molecules such as Snail, Slug, matrix metallo proteinases (MMPs) have also been involved in the EMT process in solid tumor progression [30-32]. Recent studies have demonstrated the function of CAFs in modulating the EMTassociated metastasis in solid tumors [33-35]. As an example, the expression of E-cadherin was decreased in tongue cancer cells after cocultured with CAFs in vitro and in vivo [34]. Mechanistically, the CAFs can activate the EMT by regulating the TNF- $\alpha$-enhanced NF- $\kappa B / A k t$ signaling pathway [36] or by elevating secretion of MMPs to accelerate degradation of extracellular matrix (ECM) $[37,38]$.

Thy-1, also known as CD90, is a glycoprotein with a high homology to the immunoglobulin-like superfamily of cell adhesion molecules $[39,40]$. It has been reported that Thy-1 plays important roles in axon development, neuron regeneration, cell adhesion, apoptosis, inflammation and more importantly, tumor metastasis [41-43]. In pulmonary fibroblast cells, Thy-1 was reported to promote cell proliferation by affecting cytokines [44]. Thy-1 knockout resulted in drug-induced severe pulmonary fibrosis in Thy-1 deficient mice [45]. In contrast, Thy- 1 was found to inhibit tumor proliferation by inducing apoptosis in ovarian cancer cells [46]. However, the expression of Thy1 in CAFs and its role in CAFs-induced cancer cell EMT have not been well elucidated.

The present study was undertaken to establish the role of Thy-1

*Corresponding authors: Zhenggang Zhu, Department of General Surgery, Ruijin Hospital, Shanghai Jiao Tong University School of Medicine, Shanghai 200025, P. R. China, Tel: +86-21-64458887; Fax: +86-21-64458887; E-mail: rjzzg@sina.com

Minhua Zheng, Department of General Surgery, Ruijin Hospital, Shanghai Jiao Tong University School of Medicine, Shanghai 200025, P. R. China, Tel: +86-2164458887; Fax: +86-21-64458887; E-mail: zmhtiger@yeah.net

Received August 28, 2014; Accepted September 22, 2014; Published September 26, 2014

Citation: Hu W, Li C, Sun J, Feng B, Zhang D, et al. (2014) Cancer-AssociatedFibroblast Induces Epithelial-Mesenchymal Transition of Gastric Cancer Cells via Activating Thy-1. J Carcinog Mutagen 5: 190. doi:10.4172/2157-2518.1000190

Copyright: (c) $2014 \mathrm{Hu}$ W, et al. This is an open-access article distributed under the terms of the Creative Commons Attribution License, which permits unrestricted use, distribution, and reproduction in any medium, provided the original author and source are credited. 
expressed in CAFs in the CAFs-induced EMT and gastric cancer progression. We showed that the CAFs isolated from gastric tumors induce EMT and cancer cell aggressiveness, and the enhanced Thy1 expression in CAFs is a key effector. Our studies demonstrate a significant role and a novel mechanism of CAFs in promoting EMTinduced metastasis in human gastric cancer.

\section{Materials and Methods}

\section{Patients enrollment and ethic statements}

Patients (54 in total) who underwent surgical treatment for gastric cancer were enrolled from December 2010 until April 2012 in Shanghai Minimally Invasive Surgery Center, Ruijin Hospital, Shanghai Jiao Tong University School of Medicine. All experiments were conducted in accordance with the guidelines of the National Institutes of Health for the Care. The study protocol was also approved through the Ethical Committee on the Use of Human Tissues in Teaching and Research, Ruijin Hospital, Shanghai Jiao Tong University School of Medicine and the written informed consents were obtained from all patients involved prior to this study. None of the patients enrolled had been accepted preoperative radiotherapy or chemotherapy. All patients enrolled were diagnosed with gastric cancer after postoperative pathologic examination. Exclusion criteria were: in situ disease or malignant disease in the past 5 years (except superficial squamous or basal cell carcinoma of the skin or in situ cervical cancer).

\section{Cell lines and cell culture}

The primary cultures of CAF and paired normal fibroblast (NF) were obtained as described previously [35,47]. Briefly, the primary tumor was excised and minced under aseptic conditions. The tumor pieces were cultivated in RPMI 1640 (Invitrogen, Grand Island, NY) with $10 \%$ heat-inactivated fetal calf serum (FCS; Hyclone, Logan, UT), $100 \mu \mathrm{g} / \mathrm{ml}$ penicillin/ streptomycin (Invitrogen), and $0.5 \mathrm{mM}$ sodium pyruvate (Invitrogen), and incubated in humidified incubators at $37^{\circ} \mathrm{C}$ with $5 \% \mathrm{CO} 2$. After $\sim 2$ weeks of incubation, the fibroblasts were collected and transferred to another culture dish. Serial passages were then carried out for every 4-7 days. The fibroblasts were used 3-12th generations in culture. Paired NF was prepared from the normal gastric tissues excised at the resection margin of each case (at least $>5 \mathrm{~cm}$ away from the cancer tissue and pathologically confirmed as normal gastric tissue) following the same procedure. Human gastric cancer cell line MKN28 was obtained from the American Type Culture Collection (Manassas, VA) and were grown under established conditions.

\section{Transwell cell migration and invasion assay}

The transwell migration and invasion assay was performed using the CytoSelect 24-Well Cell Migration and Invasion Assay kit (Cell Biolabs, San Diego, CA) as described previously [27,29]. For co-culture investigations, the CAF cells or the paired NF cells were counted and implanted into the 24 -well plate with a density of $4 \times 104 /$ well. The MKN 28 gastric cancer cells with a density of $1 \times 105 /$ well were placed into the upper chamber. After 48 hours of incubation at $37^{\circ} \mathrm{C} / 5 \% \mathrm{CO} 2$, the migratory/ invasive cells passed through basement membrane (matrix protein layer and/or polycarbonate membrane) and clung to the bottom of the insert. Upon removal of cells within inserts with cotton swabs, migratory/invasive cells were stained and quantified at $550 \mathrm{~nm}$, according to the provided protocol.

\section{Thy-1 silencing by small interfering RNA}

Knockdown of Thy-1 expression by small interfering RNA (siRNA) was performed according to the manufacturer's instructions. Briefly, the CAF cells at $\sim 60 \%-70 \%$ confluence were transfected with either Thy-1 siRNA (si-Thy-1, Genepharma, Shanghai, China) or the control siRNA (si-Control) at $10 \mathrm{nM}$ for 24 hours, using transfection reagent Lipofectamine 2000 (Invitrogen). The efficiency of Thy-1 knockdown was examined by Western blot.

\section{Real-time PCR}

Total RNA was extracted from cells and tissues using Trizol (Invitrogen) according to the manufacturer's protocol. Total RNA (0.5 $\mu \mathrm{g}$ ) from each sample was used for first-strand cDNA synthesis using a Plexor ${ }^{\oplus}$ Two-Step qRT-PCR System (Promega, WI). The quantitative real-time (qRT)-PCR was carried out using the cDNA as a template and Universal PCR Master Mix (Applied Biosystems, Carlsbad, CA) on a $7900 \mathrm{HT}$ sequence detection system (Applied Biosystems). Primers used for qRT-PCR analysis were as follows: $\alpha$-SMA , CAGGG CTGTT TTCCC ATCC AT (forward) and GCCAT GTTCT ATCGG GTACT TC (reverse); Thy-1, 5'-TCAGG AAATG GCTTT TCCCA-3' (forward) and 5'-TCCTC AATGA GATGC CATAA GCT-3' (reverse); GAPDH, 5'-CCTCA ACGAC CACTT TGTCA-3' (forward) and 5'-TTACT CCTTG GAGGC CATGT-3' (reverse). GAPDH was used as a loading control. Data were expressed as a relative amount of mRNA normalized to GAPDH.

\section{Protein extraction and immunoblotting}

Preparation of cell lysates and immunoblot analysis were performed as previously described [27,29]. Primary antibodies used include: E-cadherin (\# 5296), Vimentin (\# 3390), MMP-9 (\# 13667), Snail (\# 3879) and Slug (\# 9585) from Cell Signaling Technology (Beverly, MA); Thy-1 (ab92574) from Abcam (Cambridge, MA); GAPDH (sc-32233) from Santa Cruz Biotechnology (Santa Cruz, CA). The secondary antibodies including horseradish peroxidase-conjugated anti-rabbit (A6154) or anti-mouse (A4416) antibodies were supplied from SigmaAldrich (St. Louise, MA). The chemiluminescent signals were detected using SuperSignal West Pico Chemiluminescent Substrate (Pierce, Thermo Fisher Scientific, Rockford, IL).

\section{Immunohistochemistry (IHC)}

Tumor tissues and the paired non-tumor tissues at the resection margins were collected, fixed with formaldehyde and embedded with paraffin as per standard protocol. IHC staining was performed as previously described [48]. In brief, after permeabilization and antigen-retrieval, the sections were incubated with anti-Thy-1 antibody (ab92574, Abcam) at $40^{\circ} \mathrm{C}$ overnight, followed by incubation for $1 \mathrm{~h} /$ RT with the horseradish peroxidase-conjugated secondary antibody (A6154, Sigma-Aldrich). For the negative controls, the blocking peptide was applied to the section prior to probing with primary antibody. The sections were then washed with TBS and treated with the 2-Solution DAB Kit (Invitrogen, Camarillo, CA) according to the manufacturer's procedure. The tissues were counterstained with Mayer's hematoxylin. All the IHC sections were examined and scored under a light microscope (Olympus, Tokyo, Japan) by a pathologist and the principal researchers. Cases with discrepant scores were rescored by the same or additional scorers to obtain a consensus score. Thy-1 scoring was done according to the widely-used German semiquantitative scoring system, taking into account the staining intensity and the percentage of stained tumor cells. Staining levels were scored as 0 (no staining), 1 (weak staining), 2 (moderate staining) and 3 (strong staining), based on the staining intensity in the tumor cells. The percentage of stained tumor cells in each section was counted and the sections were scored accordingly $(<10 \%=0,10-25 \%=1,26-50 \%=2,51-$ 
Citation: Hu W, Li C, Sun J, Feng B, Zhang D, et al. (2014) Cancer-Associated-Fibroblast Induces Epithelial-Mesenchymal Transition of Gastric Cancer Cells via Activating Thy-1. J Carcinog Mutagen 5: 190. doi:10.4172/2157-2518.1000190

$75 \%=3,76-100 \%=4)$. The final immunostainning score of each tumor tissue section was determined by multiplying the intensity scores with the scores of positively stained tumor cells, with the minimum score of 0 and a maximum score of 12 . Tumor sections with score $1-4$ were considered as negative, whereas tumor sections with score 5-12 were considered as positive.

\section{Statistical analysis}

Data were expressed as mean \pm standard deviation (SD) from at least 3 independent experiments. One-way ANOVA or the Student's $\mathrm{t}$ test was used for statistically analysis. Results were considered as significant when $\mathrm{p}<0.05$.

\section{Results}

\section{Identification and characterization of CAF and paired NF in gastric cancer}

CAFs have been demonstrated to play critical roles in the remodeling of ECM in solid tumor and this tissue structural change promotes invasion and metastasis [49-51]. Compared to the NFs, the CAFs in the tumor microenvironment potentially develop functional changes to cooperate with the tumor cells or other stromal cells
[35], and therefore, is considered as activated fibroblasts [19,21,37]. In particular, $\alpha$-SMA is highly expressed in the CAFs and used as a marker for the identification and characterization of CAFs [52-54]. In this study, we isolated the CAF cells and the matched NF cells from 5 pairs of gastric tumor and non-tumor tissues and the expression of a-SMA was examined.

Morphological analysis showed that the CAF cells isolated from gastric tumors are quite similar to the paired NF cells (Figure 1A). The CAF cells are mainly spindle, with a few cells showing triangle and/or polygon phenotype. When re-implanted in a new dish, the CAF cells are characterized as pseudopodia with short mitotic cycle. Most of the CAF cells are stretched and polarized with a flat long fusiform shape. The CAF cells can interlace into a reticular structure in low cell density and fuse into bundles at full confluence.

The expression of $\alpha$-SMA mRNA, a biomarker of CAF cells, was analyzed by real-time PCR in the CAF cells and the paired NF cells. As indicated in Figure 1B, the CAF cells from the 5 tumors showed a significantly increased expression of $a$-SMA mRNA comparing to the paired NF cells $(\mathrm{p}<0.001)$. Therefore, the isolated fibroblast cells from the gastric tumor tissues are typical CAF cells based on the cell morphology and the abnormal high expression of a-SMA mRNA.

(A)

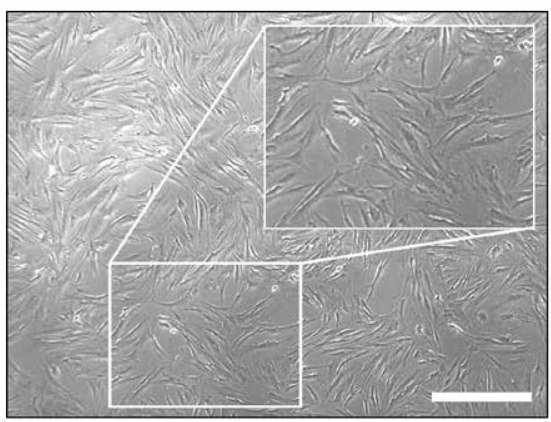

Gastric cancer-associated fibroblast

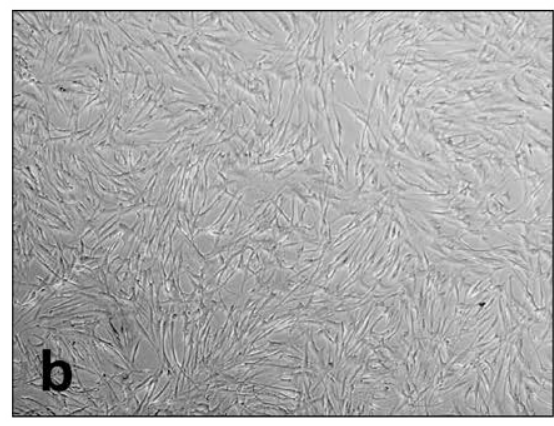

Paired normal fibroblast

(B)

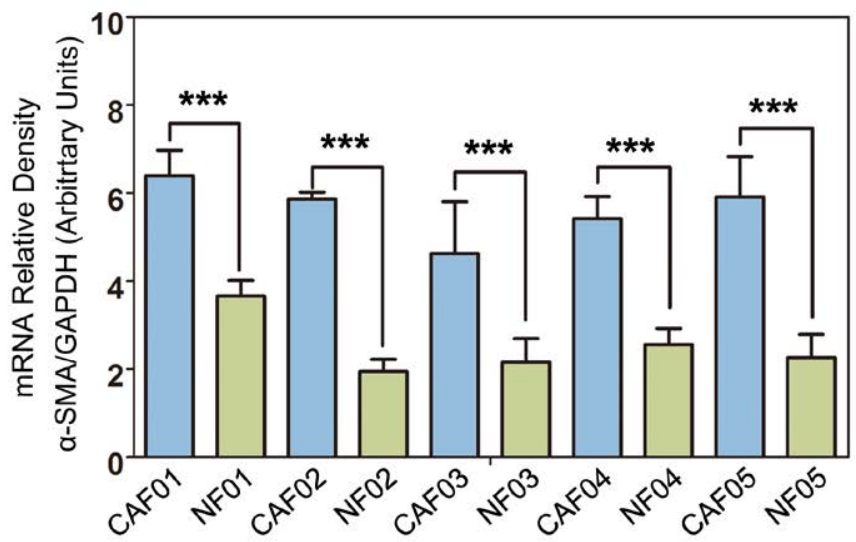

Figure 1: Gastric cancer-associated fibroblasts (CAFs) and paired normal fibroblasts (NFs). (A) Representative morphology of the CAF cells (a: CAF02) and paired NF cells (b: NF02). The morphology of CAF cells in gastric cancer is quite similar to the paired NF cells, i.e., polarized spindle shape, clear boundary, good stereoscopic effects and high refractive index, with a few cells showing triangle and/or polygon in shape. Scale bars: $100 \mu \mathrm{m}$. (B) Real-time PCR analysis for a-SMA mRNA expression in the isolated CAF cells and paired NF cells from 5 specimens. The results indicated that in all 5 gastric tumors, the CAF cells showed 1.7- to 3.0-fold increase of $\alpha$-SMA mRNA expression comparing to the paired NF cells. ${ }^{* \star *}, p<0.001$, relative to the paired NF cells. 
Citation: Hu W, Li C, Sun J, Feng B, Zhang D, et al. (2014) Cancer-Associated-Fibroblast Induces Epithelial-Mesenchymal Transition of Gastric Cancer Cells via Activating Thy-1. J Carcinog Mutagen 5: 190. doi:10.4172/2157-2518.1000190

\section{Cafs induce EMT and EMT-associated migration and invasion in co-cultured gastric cancer cells}

To further investigate the effects of CAFs on gastric cancer, the 5 CAFs clones and paired NF clones were, respectively, co-cultured with MKN28 gastric cancer cells and the migration and invasion of the co-cultured MKN28 cells were assessed using the transwell assay. As indicated in Figure 2A, MKN28 cells co-cultured with CAF cells showed a significant increase of both migratory $(\mathrm{p}<0.001)$ and invasive capacities $(\mathrm{p}<0.001)$ compared with the cells co-cultured with $\mathrm{NF}$ cells (Figure 2A). These results suggest that CAFs markedly promote migration and invasion of gastric cancer cells.

Epithelial-mesenchymal transition (EMT) is now regarded as the key process to induce cancer cell migration, invasion and metastasis. We then investigate whether the CAFs-enhanced cancer cell migration and invasion is associated with EMT. To this end, the expression of the EMT markers including E-cadherin and vimentin, and the EMTassociated key molecules such as Snail, Slug and MMP-9, were evaluated in MKN28 cells co-cultured with CAFs or NFs. In comparison with the NFs, co-culturing with the CAFs significantly inhibited the expression of E-cadherin $(\mathrm{p}<0.001)$ in MKN28 cells (Figure $2 \mathrm{~B})$. In contrast, the CAFs markedly elevated the expression of vimentin, Snail, Slug and
MMP-9 in MKN28 cells, relative to the NFs $(\mathrm{p}<0.05-0.001)$ (Figure 2B). These data indicate that the CAFs are able to drive EMT in gastric cancer cells.

\section{Thy-1 expression is elevated in gastric CAFs}

Accumulating evidences support that CAFs have functions in both tumor progression and carcinogenesis [41,46]. Thy-1 is a glycosylphosphatidylinositol (GPI)-anchored protein (25-37 kDa) involved in $\mathrm{T}$ cell activation, neuron growth, apoptosis, tumor progression, wound healing, and fibrosis [46]. These diverse effects attribute to its participation in multiple signaling cascades [55]. Its functions in cancer progression have not been well understood. Recent studies have demonstrated that carcinomas, regardless of Gleason grade, showed a layer of Thy-1 positive stromal fibroblastic cells, $\sim 5$ to 10 cells deep in tumor glands, as assessed by immunohistochemistry [56]. However, few studies have been reported regarding to the mechanism of Thy-1 in gastric cancer progression and metastasis. Because the CAFs have functions in both tumor progression and carcinogenesis and our studies further proved the role of CAFs in facilitating gastric cancer cell aggressiveness (Figure 2), we aimed to understand if Thy-1 plays a critical role in the CAFs-driven gastric cancer metastasis.

In human gastric tumors, Thy-1 expression examined by IHC
(A)
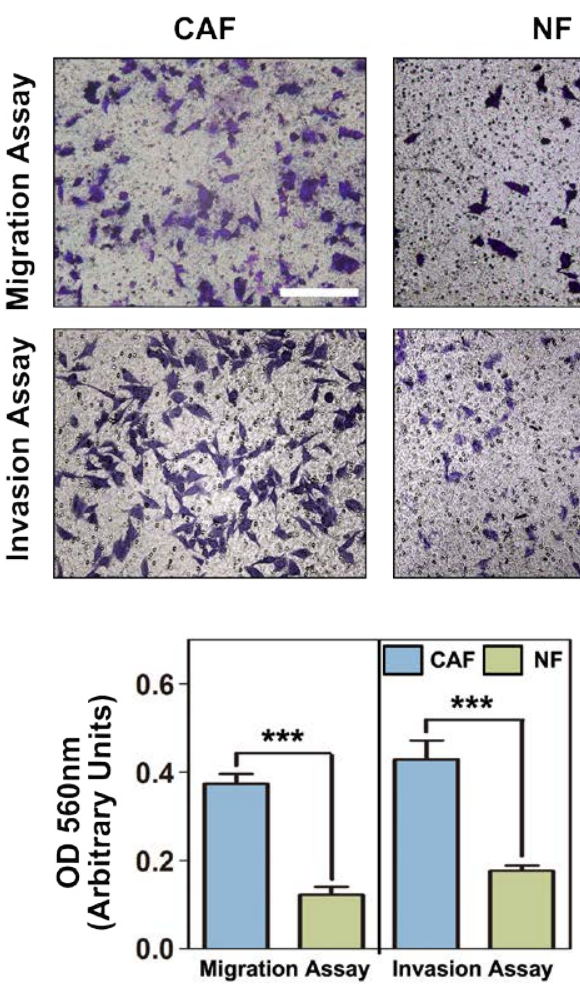

(B)
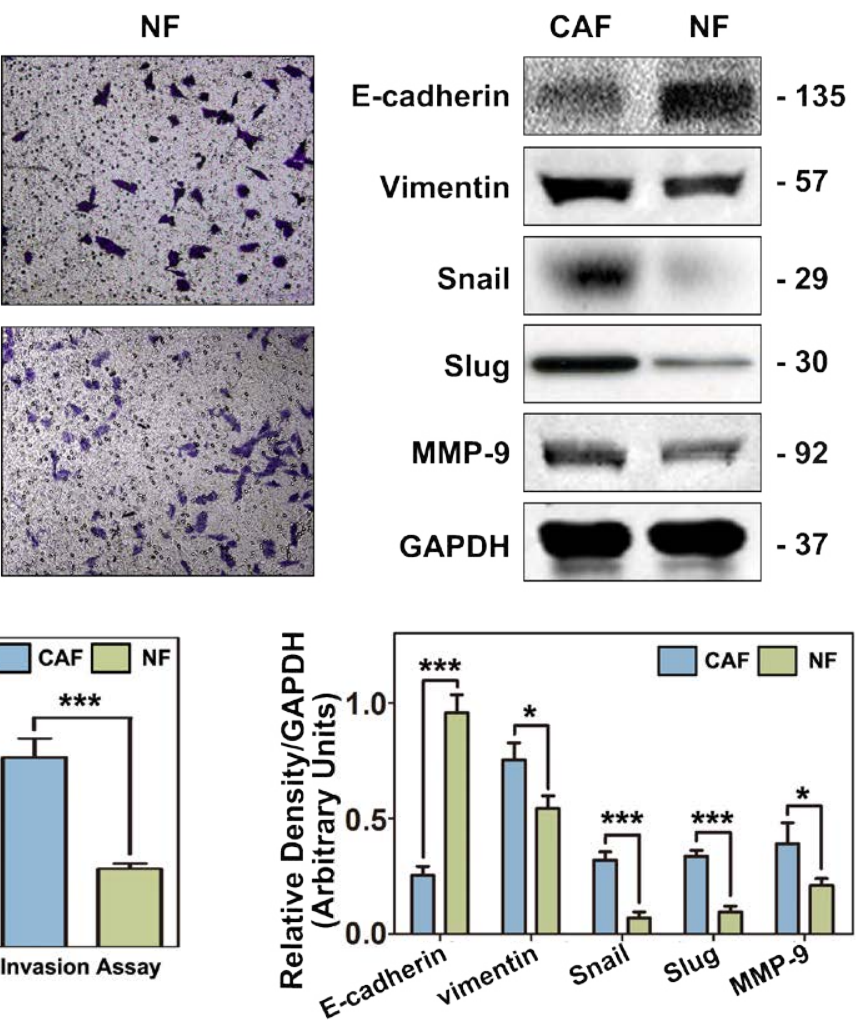

Figure 2: CAFs Promotes EMT and EMT-associated Migration and Invasion in Co-cultured MKN28 Gastric Cancer Cells. (A) Transwell migration and invasion of MKN28 gastric cancer cells co-cultured with CAF or NF cells. These figures showed the representative results from CAF02 and the paired NF02 cells. When cocultured with the CAF cells, the MKN28 gastric cancer cells showed a significant increase of migratory and invasive capacities compared with the cells co-cultured with the NF cells. Scale bar: $200 \mu \mathrm{m}$. ${ }^{* * *}, \mathrm{p}<0.001$, relative to the MKN28 cells co-cultured with the paired NF cells. (B) Expressions of EMT markers in MKN28 cells after co-cultivation. In MKN28 gastric cancer cells, the expression of E-cadherin was significantly increased by $>4$-folds when co-cultured with the CAF cells, compared to the cells co-cultured with the NF cells. On the contrary, relative to the NF cells, co-culturing with the CAF cells resulted in significantly decreases of the expression of vimentin, Snail, Slug and MMP-9 (by $\sim 28 \%, \sim 78 \%, \sim 71 \%$ and $\sim 46 \%$, respectively) in MKN28 cells. ${ }^{*}$, p<0.05; ${ }^{* * *}$, p<0.001, relative to the cells cocultured with the NF cells. 
Citation: Hu W, Li C, Sun J, Feng B, Zhang D, et al. (2014) Cancer-Associated-Fibroblast Induces Epithelial-Mesenchymal Transition of Gastric Cancer Cells via Activating Thy-1. J Carcinog Mutagen 5: 190. doi:10.4172/2157-2518.1000190

was predominantly presented in the cytoplasm and membrane of the spindle-shaped cells among the nests of gastric carcinoma, i.e. gastric CAF cells (Figure 3A). Of the 54 gastric tumor specimens, Thy-1 expression was positive in 50 cases $(92.6 \%)$. However, in the paired normal tissues, only 12 cases $(22.2 \%)$ showed positive expression of Thy- 1 and 42 cases (77.8\%) of negative expression. This indicates that Thy- 1 expression in the CAFs may facilitate tumorigenesis in human stomach.

The increased expression of Thy-1 was further substantiated by immunoblot in the isolated CAF clones from 5 primary tumors and the paired NF clones (Figure 3B). Comparing to the paired NF clones, 4 CAF clones showed a significant increase of Thy- $1(\mathrm{p}<0.001)$. Only 1 CAF clone (CAF03) expressed a similar level of Thy- 1 to that observed in the paired NF clone (NF03). The elevated expression of Thy-1 in the CAFs was also analyzed by Real-time PCR. As indicated in Figure 3C, all the CAF clones excepting CAF03 expressed a significant elevation of Thy-1 mRNA ( $\mathrm{p}<0.001)$ compared to the paired NF clones. As such, we demonstrated that Thy-1 was over-expressed in the gastric CAF cells.

The elevated Thy-1 in CAFs regulates EMT and EMT-associated aggressiveness in Gastric Cancer

We next investigated whether the elevated Thy- 1 in the CAFs is responsible for the CAF-enhanced EMT and gastric cancer cell aggressiveness. To this end, the expression of Thy- 1 was repressed in the CAFs (e.g., CAF02 and CAF04) by treating with the Thy-1-specific siRNA. The CAF cells treated with scramble control siRNA were used as control. These CAF cells were then co-cultured with MKN28 cells to evaluate the effect of Thy-1 depletion on cancer cell migration/ invasion. We showed that treatment of Thy-1 siRNA efficiently reduced Thy- 1 expression in these CAFs clones ( $\mathrm{p}<0.001$, (Figure $4 \mathrm{~A}$ ). Depletion of Thy- 1 in these CAFs resulted in a significant reduction of both migratory and invasive capacities in MKN28 cells comparing to the CAF cells treated with control siRNA $(\mathrm{p}<0.001)$ (Figure 4B). These findings suggest that Thy-1 expression in CAFs is a critical factor in promoting aggressive behavior of gastric cancer cells.

We also evaluated the expressions of EMT markers in MKN28 cells after co-cultured with the CAF cells treated with Thy-1 siRNA or control siRNA (Figure 4C). The results showed that the expression of the epithelial marker E-cadherin was markedly increased (3- to 6 -fold, $\mathrm{p}<0.001$ ), while the expression of the mesenchymal markers, in particular, vimentin, was significantly reduced $(42-95 \%, p<0.001)$ in MKN28 cells co-cultured with Thy-1-knowckdown CAF cells compared to the cells co-cultured with control siRNA-treated CAF cells. In addition, relative to the control siRNA-treated CAF cells, the
(A)
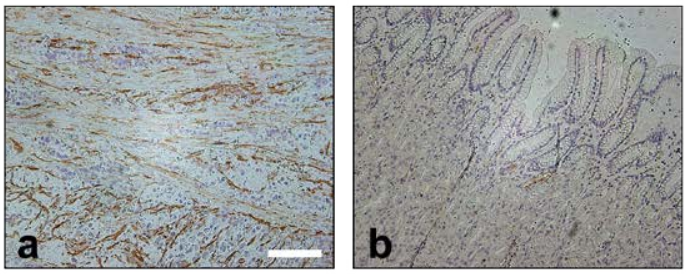

Gastric cancer tissue Normal gastric tissue
(C)

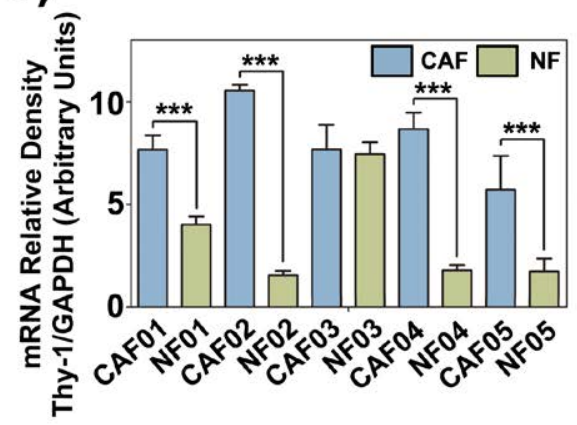

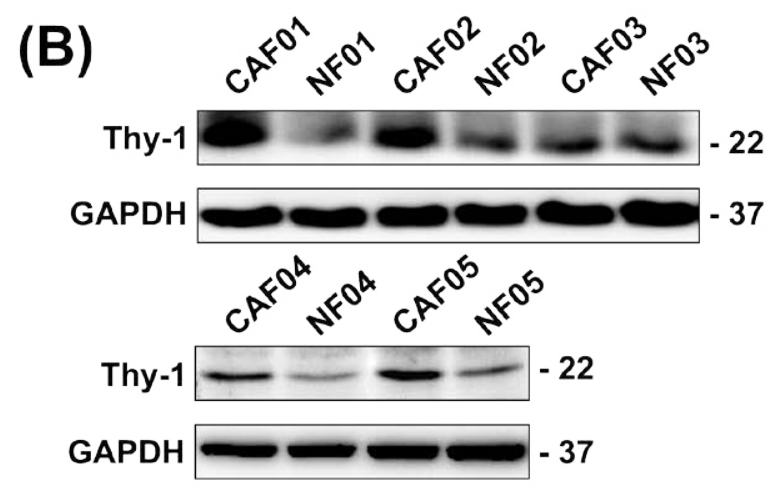

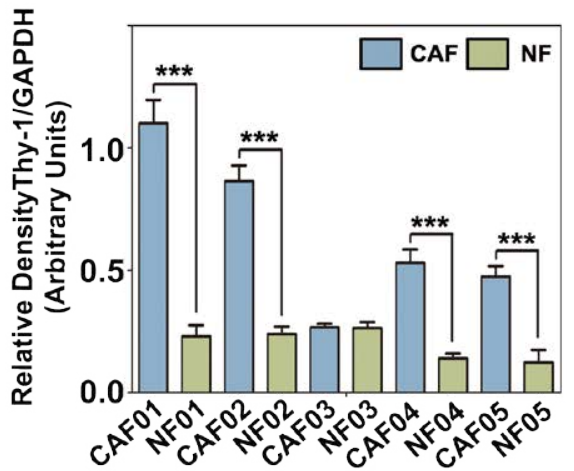

Figure 3: Thy-1 is Highly Expressed in Human Gastric Cancer-associated Fibroblast (CAF). (A) Representative immunohistochemistry staining of Thy-1 expression in gastric cancer samples. a. Thy-1 was predominantly presented in the cytoplasm and membrane of the spindle-shaped cells among the nests of gastric carcinoma, i.e. gastric CAF cells as indicated by arrows (ADD ARROWS IN THE FIGURE). b. Normal gastric tissue has low expression of Thy-1. Scale bar: 100 Mm. Magnification: 100× (BETTER TO INSERT A IMAGE WITH HIGH MAGNIFICATION). (B) Thy-1 expression in CAF clones and paired NF clones isolated from 5 gastric specimens. Note that in these 5 CAF clones, 4 CAF clones (CAF01, CAF02, CAF04 and CAF05) showed significant increase of Thy-1 protein expression relative to the paired $\mathrm{NF}$ clones, as indicated by densitometry analysis. Only 1 CAF clone (CAF03) expressed a similar level of Thy-1 to that observed in the paired NF clone (NF03). ${ }^{* * *}$, $\mathrm{p}<0.001$, relative to paired NF clones. (C) Real-time PCR analysis for Thy-1 mRNA expression. Similar to the results from the Western blot analysis, Thy-1 mRNA level was significantly up-regulated in 4 CAF clones (CAF01, CAF02, CAF04 and CAF05) compared to the paired NF clones Only the CAF03 clone expressed a similar level of Thy- 1 to that observed in the paired NF03 clone. ${ }^{* * *}, p<0.001$, relative to paired NF clones. 
(A)
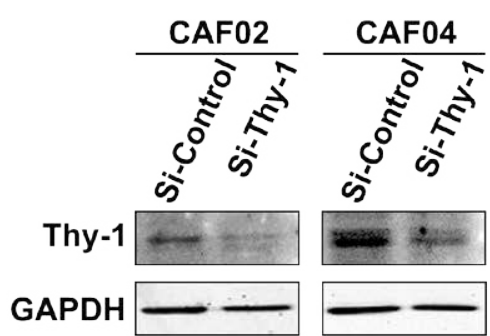

$-22$

$-37$

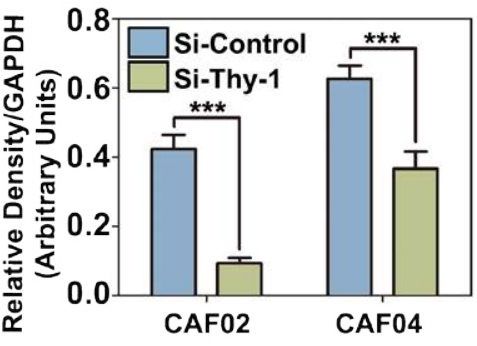

(C)

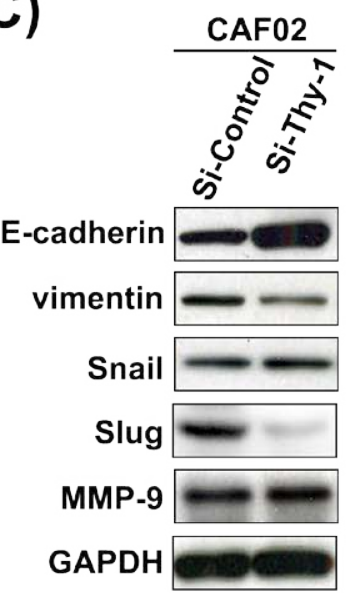

CAF04

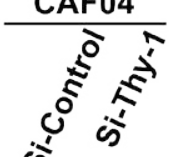

(B)
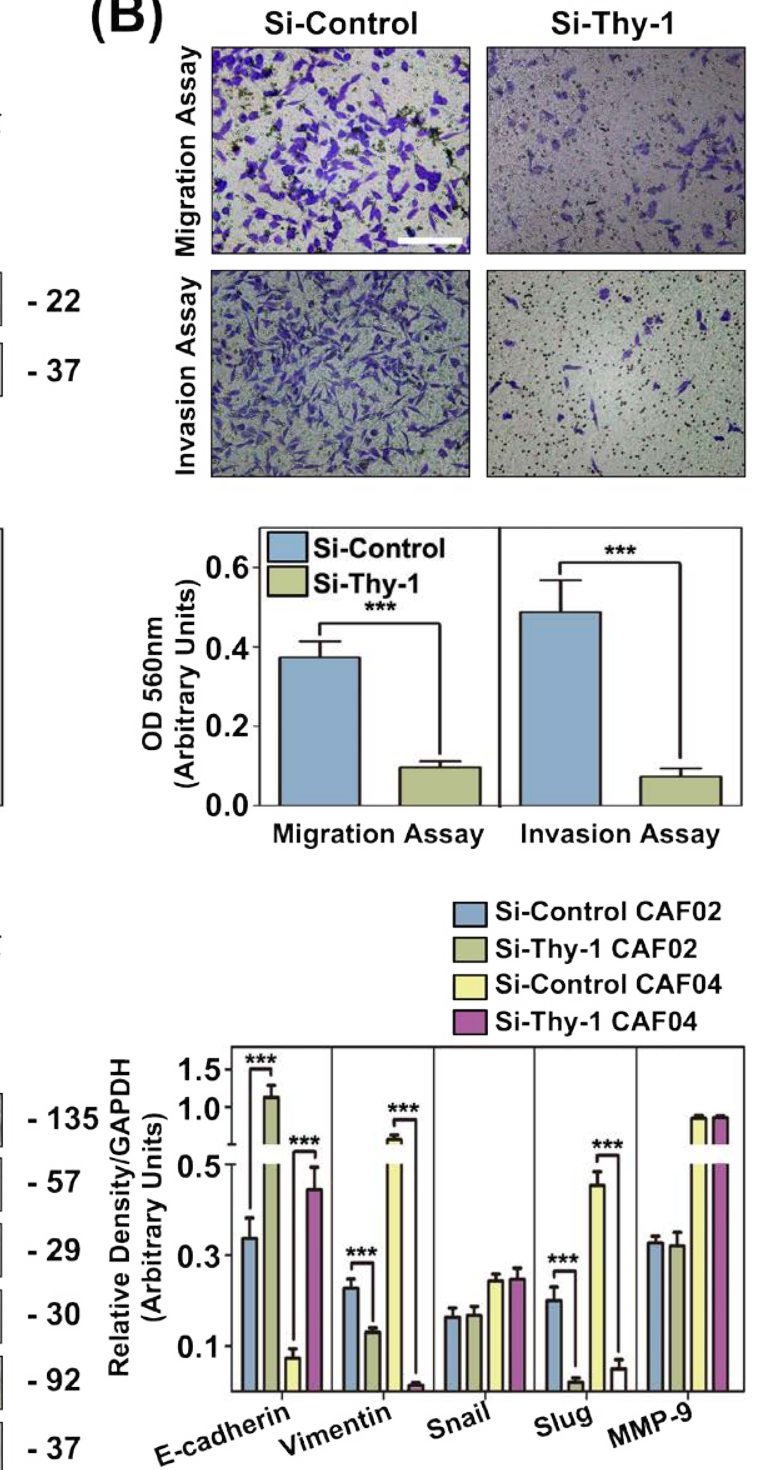

Figure 4: Thy-1 in CAFs regulates the CAF-induced EMT and EMT-associated aggressive behavior in MKN28 cancer cells. (A) Treatment with Thy-1-specific siRNA (si-Thy-1) significantly reduced Thy-1 expression in both two primary CAF clones (CAF02 and CAF04). ${ }^{* *}$, p<0.001, relative to control siRNA (si-Control). (B) Transwell migration and invasion of MKN28 cells co-cultured with the CAF cells treated with si-Thy-1 or si-Control. Cell migration and invasion were performed as described in the "Materials and Methods". Note that both the migratory and invasive capacities were markedly reduced in the MKN28 cells co-cultured with the CAF cells treated with si-Thy-1, compared to the cells co-cultured with the CAF cells treated with si-Control. Scale bar: $200 \mu$ m. ${ }^{* * *}$, $<<0.001$, relative to the CAF cells treated with si-Control. (C) Expressions of EMT markers in MKN28 cells co-cultured with the CAF cells treated with si-Thy-1 or si-Control. The results showed that expression of the epithelial marker E-cadherin was significantly increased by 3- to 6-folds in MKN28 cells after co-cultured with Thy-1 knockdown CAF cells, compared to the cells co-cultured with si-Control-treated CAF cells. In addition, after co-cultured with Thy-1 knockdown CAF cells, MKN28 cells showed a marked reduction of the expression of the mesenchymal markers, vimentin and Slug, by $\sim 42-95 \%$ and $89-90 \%$, respectively. Thy-1 knockdown in CAFs did not significantly affect the expression of Snail and MMP-9 in MKN28 cells, indicating a targeted regulation of Thy-1 for the E-cadherin transcription repressors in the CAF-induced EMT process. ${ }^{* *}, p<0.001$, relative to si-Control-treated CAF cells.

Thy-1-knowckdown CAF cells caused a $89-90 \%$ reduction of Slug, a key regulator of EMT, in MKN28 cells. Interestingly, repression of Thy1 in the CAFs had no effect on the expression of Snail and MMP-9 in the MKN28 cells. This implicates that the E-cadherin transcription repressor, slug, is a potential target of Thy-1 in modulating EMT in gastric cancer cells.

\section{Discussion}

Solid tumor cells usually invade the adjacent organs and metastasize through remodeling of the ECM [57]. In the tumor microenvironment, the CAFs are one of the major components which regulate tumor cell function by secreting growth factors, chemokines and ECM [58,59]. Therefore, CAFs play important roles in modulating solid tumor invasion and metastasis. Comparing to NFs, the CAFs underwent certain phenotypic and functional changes such as promoting cancer proliferation and chemo-sensitivity [60-62]. In addition, CAFs have also been proved to enhance cancer angiogenesis, immune cell infiltration, colony forming ability and more importantly, metastasis [62-64]. In this study, we isolated and characterized the CAF cells and the paired 
Citation: Hu W, Li C, Sun J, Feng B, Zhang D, et al. (2014) Cancer-Associated-Fibroblast Induces Epithelial-Mesenchymal Transition of Gastric Cancer Cells via Activating Thy-1. J Carcinog Mutagen 5: 190. doi:10.4172/2157-2518.1000190

NF cells from the gastric tumor and non-tumor tissues. Our studies showed that the CAFs drive EMT and facilitate cell aggressiveness in MKN28 gastric cancer cells and the increased Thy- 1 in the CAFs plays a critical role in this process.

Recently, it was reported that CAF could induce EMT in solid tumor [65-67]. As one of the important pathophysiological phenomenon, the EMT helps cancer cells to obtain the migratory and invasive abilities during metastasis in human solid tumor [65-67]. When cancer cells undergo EMT, these cells lose their epithelial characteristics such as epithelial morphology, cell polarity, cell-cell adhesiveness and gain mesenchymal properties such as fibroblastic morphology, increased migration and invasion capacities and improved metastatic activity $[66,67]$. The EMT is also characterized by the molecular changes such as loss or reduction of the epithelial surface marker E-cadherin and acquisition of the mesenchymal markers including vimentin, Snail, Slug and/or MMPs [27]. Accumulating evidence has demonstrated that when the tumor cells (e.g., colorectal, breast and lung tumors) were co-cultured with CAFs, these tumor cells acquired aggressive traits via EMT and underwent metastasis, acclaiming the prometastatic effects of CAFs $[16,17,19,38]$. In supporting of this, we also showed that the CAFs drive EMT and facilitate cell aggressiveness in MKN28 gastric cancer cells. It is thus considered that the CAFs function as "promoter" of EMT in solid tumors.

We demonstrated a significant role of Thy-1 in the CAF-regulated EMT in gastric cancer cells. As one of the glycosylphosphatidylinositol (GPI)-anchored membrane proteins, Thy- 1 is now regarded as a marker for metastasis cancer stemness in human prostate cancer, glioma, hepatocarcinoma, endometrial cancer and lung cancer [41$44,46,68-71]$. In prostate and lung cancers, Thy- 1 is up-regulated and regarded as one of the potential biomarkers for cancer treatment [56,71]. Thy- 1 is also identified as a potential stem cell marker and is regulated by the $\mathrm{Wnt} / \beta$-catenin signaling pathway in liver cancer [72]. However, only a few studies have been reported on the expression and function of Thy- 1 in CAF cells $[56,69,73,74]$. Our data indicated that Thy-1 was highly expressed not only in the human gastric cancer samples but also in the isolated CAF cells. These findings implicate a potential role of Thy-1 expressed in CAFs in the process of EMT in gastric cancer cells. Indeed, depletion of Thy-1 in CAFs decreased the capacity of migration/invasion and prevented EMT in tumor cells co-cultured with CAFs. Mechanistic studies revealed that Thy1 knockdown in CAFs markedly inhibited the expression of Slug, a transcription repressor of E-cadherin, in cancer cells. Therefore, the CAFs may promote EMT of the co-cultured gastric cancer cells by, at least, Thy-1-mediated pathway.

In summary, our studies demonstrate that gastric CAFs are able to promote EMT and migration/invasion of gastric cancer cells. The enhanced Thy- 1 expression in gastric CAFs is a key molecule in the CAFs-mediated EMT process in gastric cancer metastasis. While more studies are needed to elucidate the potential signaling pathways regulated by Thy-1, targeting Thy-1 in CAFs might be a new therapeutic strategy to prevent gastric cancer metastasis.

\section{References}

1. DeSantis CE, Lin CC, Mariotto AB, Siegel RL, Stein KD, et al. (2014) Cancer treatment and survivorship statistics, 2014. CA Cancer J Clin 64: 252-271.

2. Jung K W, Won YJ, Kong HJ, Oh CM, Lee DH, et al. (2014) Cancer statistics in Korea: incidence, mortality, survival, and prevalence in 2011. Cancer Res Treat 46: 109-123

3. Siegel R, Ma J, Zou Z, Jemal A (2014) Cancer statistics, 2014. CA: a cancer journal for clinicians 64: 9-29
4. Rocco A, Compare D, Nardone, G (2012) Cancer stem cell hypothesis and gastric carcinogenesis: Experimental evidence and unsolved questions. World journal of gastrointestinal oncology: 54-59

5. Saikawa Y, Fukuda K, Takahashi T, Nakamura R, Takeuchi H, et al. (2010) Gastric carcinogenesis and the cancer stem cell hypothesis. Gastric cancer official journal of the International Gastric Cancer Association and the Japanese Gastric Cancer Association 13: 11-24

6. Xu G, Shen J, Ou Yang X, Sasahara M, Su X (2013) Cancer stem cells: the 'heartbeat' of gastric cancer. J Gastroenterol 48: 781-797.

7. Kharaishvili G, Simkova D, Bouchalova K, Gachechiladze M, Narsia N, et al. (2014) The role of cancer-associated fibroblasts, solid stress and other microenvironmental factors in tumor progression and therapy resistance. Cancer cell international 14: 41

8. Lisanti M P, Martinez-Outschoorn U E, Sotgia F (2013) Oncogenes induce the cancer-associated fibroblast phenotype: metabolic symbiosis and "fibroblast addiction" are new therapeutic targets for drug discovery. Cell cycle 12: 27232732

9. Luga V, Wrana J L (2013) Tumor-stroma interaction: Revealing fibroblastsecreted exosomes as potent regulators of Wnt-planar cell polarity signaling in cancer metastasis. Cancer research 73: 6843-6847

10. Hasegawa T, Yashiro M, Nishii T, Matsuoka J, Fuyuhiro Y, et al. (2014) Cancer-associated fibroblasts might sustain the stemness of scirrhous gastric cancer cells via transforming growth factor-beta signaling. International journal of cancer. Journal international du cancer 134: 1785-1795

11. Hu C, Wang Z, Zhai L, Yang M, Shan L, et al. (2013) Effects of cancerassociated fibroblasts on the migration and invasion abilities of SGC-7901 gastric cancer cells. Oncol Lett 5: 609-612.

12. Wang RF, Zhang LH, Shan LH, Sun WG, Chai CC, et al. (2013) Effects of the fibroblast activation protein on the invasion and migration of gastric cancer. Experimental and molecular pathology: 350-356

13. Zhang Q, Wang M, Huang F, Yang T, Cai J, et al. (2013) H. pylori infectioninduced MSC differentiation into CAFs promotes epithelial-mesenchymal transition in gastric epithelial cells. International journal of molecular medicine 32: $1465-1473$

14. Henriksson ML, Edin S, Dahlin AM, Oldenborg PA, Öberg Å, et al. (2011) Colorectal cancer cells activate adjacent fibroblasts resulting in FGF1/FGFR3 signaling and increased invasion. Am J Pathol 178: 1387-1394.

15. Herrera A, Herrera M, Alba-Castellon L, Silva J, Garcia V, et al. (2014) Protumorigenic effects of Snail-expression fibroblasts on colon cancer cells. International journal of cancer. Journal international du cancer 134: 2984-2990

16. Herrera M, Islam AB, Herrera A, Martin P, Garcia V, et al. (2013) Functiona heterogeneity of cancer-associated fibroblasts from human colon tumors shows specific prognostic gene expression signature. Clinical cancer research : an official journal of the American Association for Cancer Research 19: 5914-5926

17. Nakagawa H, Liyanarachchi S, Davuluri RV, Auer H, Martin EW, et al. (2004) Role of cancer-associated stromal fibroblasts in metastatic colon cancer to the liver and their expression profiles. Oncogene 23: 7366-7377

18. Angelucci C, Maulucci G, Lama G, Proietti G, Colabianchi A, et al. (2012) Epithelial-stromal interactions in human breast cancer: effects on adhesion, plasma membrane fluidity and migration speed and directness. PloS one 7 : e50804

19. Fu Z, Song P, Li D, Yi C, Chen $\mathrm{H}$, et al. (2014) Cancer-associated fibroblasts from invasive breast cancer have an attenuated capacity to secrete collagens. Int J Oncol 45: 1479-1488.

20. Gao MQ, Kim BG, Kang S, Choi YP, Yoon JH, et al. (2013) Human breast cancer-associated fibroblasts enhance cancer cell proliferation through increased TGF-Ît cleavage by ADAM17. Cancer Lett 336: 240-246.

21. Tchou J, Conejo-Garcia J (2012) Targeting the tumor stroma as a nove treatment strategy for breast cancer: shifting from the neoplastic cell-centric to a stroma-centric paradigm. Adv Pharmacol 65: 45-61.

22. Giannoni E, Bianchini F, Masieri L, Serni S, Torre E, et al. (2010) Reciprocal activation of prostate cancer cells and cancer-associated fibroblasts stimulates epithelial-mesenchymal transition and cancer stemness. Cancer research 70 : 6945-6956

23. Paland N, Kamer I, Kogan-Sakin I, Madar S, Goldfinger N, et al. (2009) 
Citation: Hu W, Li C, Sun J, Feng B, Zhang D, et al. (2014) Cancer-Associated-Fibroblast Induces Epithelial-Mesenchymal Transition of Gastric Cancer Cells via Activating Thy-1. J Carcinog Mutagen 5: 190. doi:10.4172/2157-2518.1000190

Differential influence of normal and cancer-associated fibroblasts on the growth of human epithelial cells in an in vitro cocultivation model of prostate cancer. Mol Cancer Res: 1212-1223

24. Korc M (2007) Pancreatic cancer-associated stroma production. Am J Surg 194: S84-86

25. Yu J, Walter K, Omura N, Hong SM, Young A, et al. (2012) Unlike pancreatic cancer cells pancreatic cancer associated fibroblasts display minimal gene induction after 5-aza-2'-deoxycytidine. PloS one 7: e43456

26. Koten JW, Den Otter W (1991) The transition of benign to malignant in epithelial and mesenchymal tumours. Anticancer Res 11: 567-568.

27. Chen Z, Zhang D, Yue F, Zheng M, Kovacevic Z, et al. (2012) The iron chelators Dp44mT and DFO inhibit TGF-beta-induced epithelial-mesenchymal transition via up-regulation of N-Myc downstream-regulated gene 1 (NDRG1) J Biol Chem 287: 17016-17028

28. Jin R, Liu W, Menezes S, Yue F, Zheng M, et al. (2014) The metastasis suppressor NDRG1 modulates the phosphorylation and nuclear translocation of $\hat{i}^{2}$-catenin through mechanisms involving FRAT1 and PAK4. J Cell Sci 127: 3116-3130.

29. Sun J, Zhang D, Bae DH, Sahni S, Jansson P, et al. (2013) Metastasis suppressor, NDRG1, mediates its activity through signaling pathways and molecular motors. Carcinogenesis 34: 1943-1954.

30. Kalluri R, Weinberg RA (2009) The basics of epithelial-mesenchymal transition J Clin Invest 119: 1420-1428.

31. Yu M, Bardia A, Wittner BS, Stott SL, Smas ME, et al. (2013) Circulating breast tumor cells exhibit dynamic changes in epithelial and mesenchymal composition. Science 339: 580-584

32. Zhang Q, Bai X, Chen W, Ma T, Hu Q, et al. (2013) Wnt/beta-catenin signaling enhances hypoxia-induced epithelial-mesenchymal transition in hepatocellular carcinoma via crosstalk with hif-1alpha signaling. Carcinogenesis 34: 962-973

33. Choe C, Shin YS, Kim SH, Jeon MJ, Choi SJ, et al. (2013) Tumor-stromal interactions with direct cell contacts enhance motility of non-small cell lung cancer cells through the hedgehog signaling pathway. Anticancer research 33 3715-3723

34. Vered M, Dayan D, Yahalom R, Dobriyan A, Barshack I, Bello I O, et al (2010) Cancer-associated fibroblasts and epithelial-mesenchymal transition in metastatic oral tongue squamous cell carcinoma. International journal of cancer. Journal international du cancer 127: 1356-1362

35. Zhou B, Chen WL, Wang YY, Lin ZY, Zhang DM, et al. (2014) A role for cancerassociated fibroblasts in inducing the epithelial-to-mesenchymal transition in human tongue squamous cell carcinoma. Journal of oral pathology \& medicine 43: $585-592$

36. Wang H, Wang HS, Zhou BH, Li CL, Zhang F, et al. (2013) Epithelialmesenchymal transition (EMT) induced by TNF- $\alpha \pm$ requires AKT/GSK-3ßmediated stabilization of snail in colorectal cancer. PLoS One 8: e56664.

37. Cai J, Tang H, Xu L, Wang X, Yang C, et al. (2012) Fibroblasts in omentum activated by tumor cells promote ovarian cancer growth, adhesion and invasiveness. Carcinogenesis 33: 20-29.

38. Gao M Q, Kim BG, Kang S, Choi YP, Park H, et al. (2010) Stromal fibroblasts from the interface zone of human breast carcinomas induce an epithelialmesenchymal transition-like state in breast cancer cells in vitro. Journal of cell science 123: 3507-3514

39. Bradley JE, Ramirez G, Hagood JS (2009) Roles and regulation of Thy-1, a context-dependent modulator of cell phenotype. Biofactors 35: 258-265.

40. Lin CS, Xin ZC, Dai J, Lue TF (2013) Commonly used mesenchymal stem cell markers and tracking labels: Limitations and challenges. Histol Histopathol 28 : 1109-1116.

41. Cheng BQ, Jiang Y, Li DL, Fan JJ, Ma M (2012) Up-regulation of thy-1 promotes invasion and metastasis of hepatocarcinomas. Asian Pac J Cancer Prev 13: 1349-1353.

42. Rege TA, Hagood JS (2006) Thy-1 as a regulator of cell-cell and cell-matrix interactions in axon regeneration, apoptosis, adhesion, migration, cancer, and fibrosis. FASEB journal : official publication of the Federation of American Societies for Experimental Biology 20: 1045-1054

43. Schubert K, Gutknecht D, Köberle M, Anderegg U, Saalbach A (2013)
Melanoma cells use Thy-1 (CD90) on endothelial cells for metastasis formation. Am J Pathol 182: 266-276.

44. He Z, Wang X, Deng Y, Li W, Chen Y, et al. (2013) Epigenetic regulation of Thy1 gene expression by histone modification is involved in lipopolysaccharideinduced lung fibroblast proliferation. Journal of cellular and molecular medicine 17: $160-167$

45. Derdak S, Penney DP, Keng P, Felch ME, Brown D, et al. (1992) Differential collagen and fibronectin production by Thy 1+ and Thy 1- lung fibroblast subpopulations. Am J Physiol 263: L283-290.

46. Rege TA, Hagood, J. S. (2006) Thy-1, a versatile modulator of signaling affecting cellular adhesion, proliferation, survival, and cytokine/growth factor responses. Biochimica et biophysica acta 1763:991-999

47. Chen W J, Ho CC, Chang YL, Chen HY, Lin CA, et al. (2014) Cancerassociated fibroblasts regulate the plasticity of lung cancer stemness via paracrine signalling. Nature communications 5: 3472

48. Mao Z, Sun J, Feng B, Ma J, Zang L, et al. (2013) The metastasis suppressor, $\mathrm{N}$-myc downregulated gene 1 (NDRG1), is a prognostic biomarker for human colorectal cancer. PLoS One 8: e68206.

49. Goetz JG, Minguet S, Navarro-Lerida I, Lazcano JJ, Samaniego R, et al. (2011) Biomechanical remodeling of the microenvironment by stromal caveolin-1 favors tumor invasion and metastasis. Cell 146: 148-163

50. Gottschling S, Granzow M, Kuner R, Jauch A, Herpel E, et al. (2013) Mesenchymal stem cells in non-small cell lung cancer--different from others? Insights from comparative molecular and functional analyses. Lung Cancer 80: 19-29.

51. Zhang L, Liu J, Wang X, Li Z, Zhang X, et al. (2014) Upregulation of Cytoskeleton Protein and Extracellular Matrix Protein Induced by StromalDerived Nitric Oxide Promotes Lung Cancer Invasion and Metastasis. Current molecular medicine 14: 762-771

52. Guan J, Zhang H, Wen Z, Gu Y, Cheng Y, et al. (2014) Retinoic acid inhibits pancreatic cancer cell migration and EMT through the downregulation of IL-6 in cancer associated fibroblast cells. Cancer Lett 345: 132-139.

53. Herrera M, Herrera A, Dominguez G, Silva J, Garcia V, et al. (2013) Cancerassociated fibroblast and M2 macrophage markers together predict outcome in colorectal cancer patients. Cancer science 104: 437-444

54. Kwak Y, Lee HE, Kim WH, Kim DW, Kang SB, et al. (2014) The clinica implication of cancer-associated microvasculature and fibroblast in advanced colorectal cancer patients with synchronous or metachronous metastases. PLoS One 9: e91811.

55. Barker TH, Hagood JS (2009) Getting a grip on Thy-1 signaling. Biochim Biophys Acta 1793: 921-923.

56. True LD, Zhang H, Ye M, Huang CY, Nelson PS, et al. (2010) CD90/THY1 is overexpressed in prostate cancer-associated fibroblasts and could serve as a cancer biomarker. Modern pathology : an official journal of the United States and Canadian Academy of Pathology, Inc 23: 1346-1356

57. Catalano V, Turdo A, Di Franco S, Dieli F, Todaro M, et al. (2013) Tumor and its microenvironment: a synergistic interplay. Semin Cancer Biol 23: 522-532.

58. Kalluri R, Zeisberg M (2006) Fibroblasts in cancer. Nat Rev Cancer 6: 392-401.

59. Pietras K, Ostman A (2010) Hallmarks of cancer: interactions with the tumor stroma. Exp Cell Res 316: 1324-1331.

60. Bhowmick NA, Chytil A, Plieth D, Gorska AE, Dumont N, et al. (2004) TGF-beta signaling in fibroblasts modulates the oncogenic potential of adjacent epithelia. Science 303: 848-851.

61. Jedeszko C, Victor BC, Podgorski I, Sloane BF (2009) Fibroblast hepatocyte growth factor promotes invasion of human mammary ductal carcinoma in situ. Cancer research 69: 9148-9155

62. Orimo A, Gupta PB, Sgroi DC, Arenzana-Seisdedos F, Delaunay T, et al. (2005) Stromal fibroblasts present in invasive human breast carcinomas promote tumor growth and angiogenesis through elevated SDF-1/CXCL12 secretion. Cell 121: 335-348

63. Augsten M, Hagglof C, Olsson E, Stolz C, Tsagozis P, et al. (2009) CXCL14 is an autocrine growth factor for fibroblasts and acts as a multi-modal stimulator of prostate tumor growth. Proceedings of the National Academy of Sciences of the United States of America 106: 3414-3419 
Citation: Hu W, Li C, Sun J, Feng B, Zhang D, et al. (2014) Cancer-Associated-Fibroblast Induces Epithelial-Mesenchymal Transition of Gastric Cancer Cells via Activating Thy-1. J Carcinog Mutagen 5: 190. doi:10.4172/2157-2518.1000190

Page 9 of 9

64. Gerber PA, Hippe A, Buhren BA, Müller A, Homey B (2009) Chemokines in tumor-associated angiogenesis. Biol Chem 390: 1213-1223.

65. Blick, T, Hugo H, Widodo E, Waltham M, Pinto C, et al. (2010) Epithelial mesenchymal transition traits in human breast cancer cell lines parallel the CD44(hi/)CD24 (lo/-) stem cell phenotype in human breast cancer. Journal of mammary gland biology and neoplasia 15: 235-252

66. Kalluri R (2009) EMT: when epithelial cells decide to become mesenchymallike cells. J Clin Invest 119: 1417-1419.

67. Thiery JP, Acloque H, Huang RY, Nieto MA (2009) Epithelial-mesenchymal transitions in development and disease. Cell 139: 871-890.

68. Parry PV, Engh JA (2012) CD90 is identified as a marker for cancer stem cells in high-grade gliomas using tissue microarrays. Neurosurgery 70: N23-24.

69. Subramaniam KS, Tham ST, Mohamed Z, Woo YL, Mat Adenan NA, et al. (2013) Cancer-associated fibroblasts promote proliferation of endometrial cancer cells. PLoS One 8: e68923.
70. Sukowati CH, Anfuso B, Torre G, Francalanci P, Crocè LS, et al. (2013) The expression of CD90/Thy-1 in hepatocellular carcinoma: an in vivo and in vitro study. PLoS One 8: e76830.

71. Yan X, Luo $\mathrm{H}$, Zhou X, Zhu B, Wang $Y$, et al. (2013) Identification of CD90 as a marker for lung cancer stem cells in A549 and H446 cell lines. Oncol Rep 30: $2733-2740$.

72. Cheng BQ, Jiang $Y$, Zhu Q, Lin WG (2014) Wnt/ $\beta$-catenin aids in regulating the proliferation of hepG2 cells mediated by thy-1. Genet Mol Res 13: 5115-5127.

73. Orr B, Riddick AC, Stewart GD, Anderson RA, Franco OE, et al. (2012) Identification of stromally expressed molecules in the prostate by tag-profiling of cancer-associated fibroblasts, normal fibroblasts and fetal prostate. Oncogene 31: $1130-1142$

74. Zhao H, Peehl DM (2009) Tumor-promoting phenotype of CD90hi prostate cancer-associated fibroblasts. Prostate 69: 991-1000. 